ANNALES

POLONICI MATHEMATICI

$88.1(2006)$

\title{
Functional differential inequalities with unbounded delay
}

\author{
by Z. KAMONT and S. Kozię (Gdańsk)
}

\begin{abstract}
Classical solutions of functional partial differential inequalities with initial boundary conditions are estimated by maximal solutions of suitable problems for ordinary functional differential equations. Uniqueness of solutions and continuous dependence on given functions are obtained as applications of the comparison result. A theorem on weak functional differential inequalities generated by mixed problems is proved. Our method is based on an axiomatic approach to equations with unbounded delay. Examples of phase spaces are given.
\end{abstract}

1. Introduction. The papers of Haar [6] and Ważewski [20] initiated the theory of first order partial differential inequalities. The fundamental result, known as the Haar-Ważewski inequality, shows that a function of several variables which is of class $C^{1}$ on the Haar pyramid and satisfies a linear differential inequality can be estimated by a solution of a suitable initial value problem for an ordinary differential equation.

There exist many generalizations of the above classical result. We list some of them below.

The differential inequality may be nonlinear with respect to the unknown function and consequently, the comparison problem may be nonlinear. The assumptions on the regularity of the unknown function considered on the Haar pyramid may be weakened. Vector-valued functions satisfying systems of partial differential inequalities can be estimated by solutions of ordinary differential problems ([13, Vol. II] and [14]).

The Haar-Ważewski inequality has been extended to semiclassical solutions of nonlinear differential inequalities ([5], [17], [18]). Comparison results for generalized entropy solutions of nonlinear differential or functional differential problems are discussed in [11] and [12]. The results of those two papers are local with respect to spatial variables. Differential inequalities and suitable comparison results for initial boundary value problems are given in [4].

2000 Mathematics Subject Classification: 35R10, 34K12.

Key words and phrases: unbounded delay, initial boundary problems, phase spaces, functional differential inequalities. 
Comparison theorems global with respect to spatial variables are presented in [2] for classical solutions and in [19, Chapter VIII] for semiclassical solutions. Differential inequalities with Kamke type comparison problems can be found in [1], [14]. An interesting result on the global uniqueness of the Cauchy problem when the right hand side of the equation satisfies the Hölder condition can be found in [3]. Infinite systems of functional differential inequalities are studied in [15]. Viscosity solutions of functional differential inequalities are studied in [16]. Functional differential versions of the Haar-Ważewski inequality can be found in [8].

The monographs [8] and [19] contain an exposition of recent developments on hyperbolic differential and functional differential inequalities.

The aim of this paper is to add a new element to the above sequence of generalizations of the Haar-Ważewski inequality. We prove comparison results for functional differential inequalities with unbounded delay.

Differential inequalities find numerous applications in the theory of first order partial differential or functional differential equations, including questions such as: estimates of solutions of initial or initial boundary value problems, estimates of domains of solutions, estimates of the difference between solutions of two problems, criterions of uniqueness and continuous dependence of solutions on given functions.

We now formulate our functional differential problem. We use vectorial inequalities with the understanding that the same inequalities hold between the corresponding components. Set $\mathbb{R}_{+}=[0, \infty)$ and $\mathbb{R}_{-}=(-\infty, 0]$.

Let $a>0, r=\left(r_{1}, \ldots, r_{n}\right) \in \mathbb{R}_{+}^{n}$, and $b=\left(b_{1}, \ldots, b_{n}\right) \in \mathbb{R}^{n}$, where $b_{i}>0$ for $1 \leq i \leq n$, be given. Fix an integer $\kappa, 0 \leq \kappa \leq n$. For each $x=\left(x_{1}, \ldots, x_{n}\right) \in \mathbb{R}^{n}$ we write $x=\left(x^{\prime}, x^{\prime \prime}\right)$, where $x^{\prime}=\left(x_{1}, \ldots, x_{\kappa}\right)$ and $x^{\prime \prime}=\left(x_{\kappa+1}, \ldots, x_{n}\right)$. We have $x^{\prime}=x$ if $\kappa=n$ and $x^{\prime \prime}=x$ if $\kappa=0$. Write $c=b+r$ and define

$$
\begin{aligned}
E & =[0, a] \times\left[-b^{\prime}, b^{\prime}\right) \times\left(-b^{\prime \prime}, b^{\prime \prime}\right], \\
E_{0} & =(-\infty, 0] \times\left[-b^{\prime}, c^{\prime}\right] \times\left[-c^{\prime \prime}, b^{\prime \prime}\right], \\
\partial_{0} E & =(0, a] \times\left(\left[-b^{\prime}, c^{\prime}\right] \backslash\left[-b^{\prime}, b^{\prime}\right)\right) \times\left(\left[-c^{\prime \prime}, b^{\prime \prime}\right] \backslash\left(-b^{\prime \prime}, b^{\prime \prime}\right]\right), \\
\Omega & =E \cup E_{0} \cup \partial_{0} E, \\
D & =\mathbb{R}_{-} \times\left[0, r^{\prime}\right] \times\left[-r^{\prime \prime}, 0\right] .
\end{aligned}
$$

For a function $z: \Omega \rightarrow \mathbb{R}$ and a point $(t, x) \in E$ we define a function $z_{(t, x)}: D \rightarrow \mathbb{R}$ as follows:

$$
z_{(t, x)}(\tau, s)=z(t+\tau, x+s), \quad(\tau, s) \in D .
$$

The function $z_{(t, x)}$ is the restriction of $z$ to the set $\mathbb{R}_{-} \times\left[x^{\prime}, x^{\prime}+r^{\prime}\right] \times$ $\left[x^{\prime \prime}-r^{\prime \prime}, x^{\prime \prime}\right]$ and this restriction is shifted to the set $D$. We consider the 
nonlinear functional differential equation with unbounded delay

$$
\partial_{t} z(t, x)=f\left(t, x, z_{(t, x)}, \partial_{x} z(t, x)\right)
$$

together with the initial boundary condition

$$
z(t, x)=\varphi(t, x) \quad \text { for }(t, x) \in E_{0} \cup \partial_{0} E
$$

where $\partial_{x} z=\left(\partial_{x_{1}} z, \ldots, \partial_{x_{n}} z\right)$ and $\varphi: E_{0} \cup \partial_{0} E \rightarrow \mathbb{R}$ and $f: E \times Y \times \mathbb{R}^{n} \rightarrow \mathbb{R}$ are given functions. Here $Y$ denotes an abstract linear space satisfying suitable axioms. Elements of $Y$ are functions from $D$ into $\mathbb{R}$, and $Y$ is called a phase space for (1).

Further assumptions on $Y$ are given in Section 3. The set $D$ is such that the functional dependence in (1) is of the Volterra type.

A function $u: \Omega \rightarrow \mathbb{R}$ is called a classical solution of (1), (2) if

(i) $u$ is continuous on $E \cup \partial_{0} E$ and $u_{(0, x)} \in Y$ for $x \in[-b, b]$,

(ii) the partial derivatives $\partial_{t} u, \partial_{x} u$ exist on $E$,

(iii) $u$ satisfies (1) on $E$ and the initial boundary condition (2) holds.

Existence results for hyperbolic functional differential equations with unbounded delay can be found in [9], [10].

The paper is organized as follows. In Section 2 we prove that under natural assumptions on given functions there is a maximal solution of an initial value problem for an ordinary functional differential equation with unbounded delay and it depends continuously on the given functions. A comparison result for first order functional differential inequalities with unbounded delay is presented in Section 3. It is the main result of the paper. Uniqueness of classical solutions of problem (1), (2) and continuous dependence on given functions are obtained as applications of the comparison result. Examples of phase spaces are given in Section 3. A theorem on weak functional differential inequalities generated by (1), (2) is presented in Section 4.

Our method is based on an axiomatic approach to equations with unbounded delay.

2. Extremal solutions of initial value problems. For any metric spaces $U$ and $W$ we denote by $C(U, W)$ the class of all continuous functions defined on $U$ and taking values on $W$.

For a function $y:(-\infty, a] \rightarrow \mathbb{R}, a>0$, and $t \in(-\infty, a]$ we define $y_{t}: \mathbb{R}_{-} \rightarrow \mathbb{R}$ by $y_{t}(\tau)=y(t+\tau), \tau \in \mathbb{R}_{-}$.

In this section we consider classical solutions of the Cauchy problem for ordinary functional differential equations with unbounded delay

$$
\begin{aligned}
y^{\prime}(t) & =g\left(t, y_{t}\right), \\
y(t) & =\eta(t) \quad \text { for } t \in \mathbb{R}_{-} .
\end{aligned}
$$


where $g:[0, a] \times X \rightarrow \mathbb{R}$ and $\eta: \mathbb{R}_{-} \rightarrow \mathbb{R}$ are given functions and $X$ is an abstract linear space satisfying suitable axioms. Elements of $X$ are functions mapping $\mathbb{R}_{\text {- into }} \mathbb{R}$, and it is called a phase space for (3).

Set

$$
X_{+}=\left\{\eta \in X: \eta(t) \geq 0 \text { for } t \in \mathbb{R}_{-}\right\} .
$$

The axioms on $X$ are the following ([7]).

Assumption $H[X] . X$ is a normed linear space with the norm $\|\cdot\|_{X}$ which satisfies the conditions:

1) if $y:(-\infty, a] \rightarrow \mathbb{R}, a>0$, is a function such that $y_{0} \in X$ and $\left.y\right|_{[0, a]} \in C([0, a], \mathbb{R})$ then

(i) $y_{t} \in X$ for $t \in(0, a]$,

(ii) there are constants $H, K, L \in \mathbb{R}_{+}$independent of $y$ such that for $t \in[0, a]$

$$
\begin{aligned}
|y(t)| & \leq H\left\|y_{t}\right\|_{X}, \\
\left\|y_{t}\right\|_{X} & \leq K \max \{|y(\tau)|: \tau \in[0, t]\}+L\left\|y_{0}\right\|_{X},
\end{aligned}
$$

(iii) the mapping $t \mapsto y_{t}$ is continuous from $[0, a]$ to $X$,

2) $\left(X,\|\cdot\|_{X}\right)$ is a Banach space.

We say that the function $g$ satisfies the monotonicity condition $W_{+}$if for any $(t, w),(t, \bar{w}) \in[0, a] \times X$ such that $w(\tau) \leq \bar{w}(\tau)$ for $\tau \in \mathbb{R}_{-}$and $w(0)=\bar{w}(0)$ we have $g(t, w) \leq g(t, \bar{w})$.

Given $w \in X$, let $s w:(-\infty, a] \rightarrow \mathbb{R}$ be defined by

$$
s w(t)=w(t) \quad \text { for } t \in \mathbb{R}_{-} \quad \text { and } \quad s w(t)=w(0) \quad \text { for } t \in[0, a],
$$

and write $s_{t} w=(s w)_{t}$ where $t \in(-\infty, a]$. Denote by $\|\cdot\|_{[0, \tau]}$ the supremum norm in the space $C([0, \tau], \mathbb{R})$ where $\tau \in \mathbb{R}_{+}$.

The aim of this section is to prove that under natural assumptions on $g$ and $\eta$ there is a local maximal solution of problem (3), (4). We also prove that the maximal solutions depend continuously on given functions. To this end, we also consider the following initial value problem:

$$
\begin{aligned}
y^{\prime}(t) & =g\left(t, y_{t}\right)+\varepsilon, \\
y(t) & =\eta(t)+\eta(t, \varepsilon) \quad \text { for } t \in \mathbb{R}_{-},
\end{aligned}
$$

where $\varepsilon \geq 0$ and $\eta(\cdot, \varepsilon) \in X$ for $\varepsilon \geq 0$.

Assumption $H[\eta(\cdot, \varepsilon)]$. The family of functions $\{\eta(\cdot, \varepsilon)\}_{\varepsilon \geq 0}$ satisfies the conditions:

1) $\eta(\cdot, \varepsilon) \in X$ and $\eta(t, \varepsilon) \geq 0$ for $t \in \mathbb{R}_{-}$and $\eta(0, \varepsilon)=\varepsilon$,

2) $\eta(t, 0)=0$ for $t \in \mathbb{R}_{-}$and

$$
\lim _{\varepsilon \rightarrow 0}\|\eta(\cdot, \varepsilon)\|_{X}=0 .
$$


TheOREM 2.1. Suppose that

1) Assumptions $H[X]$ and $H[\eta(\cdot, \varepsilon)]$ hold,

2) $g \in C([0, a] \times X, \mathbb{R})$ satisfies the monotonicity condition $W_{+}$,

3) the constants $\alpha, M, d>0$ are defined by the relations

$$
\begin{aligned}
\left|g\left(t, s_{t} \eta\right)\right| & \leq M & & \text { for } t \in[0, a], \\
\left|g(t, w)-g\left(t, s_{t} \eta\right)\right| & \leq 1 & & \text { for }\left\|w-s_{t} \eta\right\|_{X} \leq d, t \in[0, a],
\end{aligned}
$$

and

$$
\alpha=\min \left\{a, \frac{d}{2 K(M+1+0.5 d)}\right\},
$$

4) the parameter $\varepsilon$ satisfies the conditions

$$
0 \leq \varepsilon \leq d / 4, \quad K \varepsilon+L\|\eta(\cdot, \varepsilon)\|_{X} \leq d / 4 .
$$

Then the Cauchy problem (5),(6) has the maximal solution $\omega(\cdot, \varepsilon)$ on the interval $(-\infty, \alpha]$,

$$
\lim _{\varepsilon \rightarrow 0} \omega(t, \varepsilon)=\omega(t) \quad \text { uniformly on }[0, \alpha],
$$

and $\omega(\cdot)$ is the maximal solution of $(3),(4)$ on $(-\infty, \alpha]$.

Proof. Define $\chi \in X$ by

$$
\chi(t)= \begin{cases}0 & \text { for } t \in(-\infty,-1] \\ t+1 & \text { for } t \in(-1,0]\end{cases}
$$

We consider the Cauchy problem

$$
\begin{aligned}
y^{\prime}(t) & =g\left(t, y_{t}\right)+\varepsilon+\xi \\
y(t) & =\eta(t)+\eta(t, \varepsilon)+\xi \chi(t) \quad \text { for } t \in \mathbb{R}_{-} .
\end{aligned}
$$

Suppose that

$$
0<\xi \leq d / 4 \text { and } K \xi+L \xi\left\|_{\chi}\right\|_{X} \leq d / 4
$$

We prove that for $\xi$ satisfying (12) there is a solution $\omega(\cdot, \varepsilon, \xi)$ of $(10),(11)$ on $(-\infty, \alpha]$. Let $B$ denote the space of all functions $y:(-\infty, \alpha] \rightarrow \mathbb{R}$ such that $y_{0} \in X$ and $\left.y\right|_{[0, \alpha]} \in C([0, \alpha], \mathbb{R})$. For $y \in B$ we define

$$
\|y\|_{B}=\left\|y_{0}\right\|_{X}+\|y\|_{[0, \alpha]} .
$$

Then $\left(B,\|\cdot\|_{B}\right)$ is a Banach space. Let $U \subset B$ denote the set of functions $y:(-\infty, \alpha] \rightarrow \mathbb{R}$ such that

(i) $y(t)=\eta(t)+\eta(t, \varepsilon)+\xi \chi(t)$ for $t \in \mathbb{R}_{-}$,

(ii) $|y(t)-y(\bar{t})| \leq(M+1+0.5 d)|t-\bar{t}|$ for $t, \bar{t} \in[0, \alpha]$.

Define a mapping $T: U \rightarrow B$ by setting, for $y \in U$,

$$
T[y](t)= \begin{cases}\eta(t)+\eta(t, \varepsilon)+\xi \chi(t) & \text { for } t \in \mathbb{R}_{-}, \\ \eta(0)+\varepsilon+\xi+\int_{0}^{t}\left[g\left(\tau, y_{\tau}\right)+\varepsilon+\xi\right] d \tau & \text { for } t \in[0, \alpha] .\end{cases}
$$


It follows from condition 1) of Assumption $H[X]$ that $y_{t} \in X$ for $t \in[0, \alpha]$ so $T$ is well defined on $U$. Suppose that $w \in X$ and

$$
\left\|w-s_{t}(\eta+\eta(\cdot, \varepsilon)+\xi \chi)\right\|_{X} \leq d / 2 .
$$

Then

$$
\begin{aligned}
\left\|w-s_{t} \eta\right\|_{X} & \leq 0.5 d+\left\|s_{t} \eta(\cdot, \varepsilon)\right\|_{X}+\left\|s_{t}(\xi \chi)\right\|_{X} \\
& \leq 0.5 d+K \varepsilon+L\|\eta(\cdot, \varepsilon)\|_{X}+K \xi+L \xi\|\chi\|_{X} \leq d, \quad t \in[0, \alpha] .
\end{aligned}
$$

We thus get, for $w \in X$ satisfying (13),

$$
|g(t, w)| \leq M+1, \quad t \in[0, \alpha] .
$$

It follows that for $y \in U$ and $t \in[0, \alpha]$ we have

$$
\begin{aligned}
& \left\|y_{t}-s_{t}(\eta+\eta(\cdot, \varepsilon)+\xi \chi)\right\|_{X} \\
& \quad \leq K \max \{|y(\tau)-(\eta(0)+\varepsilon+\xi)|: \tau \in[0, \alpha]\} \leq K(M+1+0.5 d) \alpha \leq 0.5 d
\end{aligned}
$$

and consequently

$$
|T[y](t)-T[y](\bar{t})| \leq\left|\int_{t}^{\bar{t}}\left[g\left(\tau, y_{\tau}\right)+\varepsilon+\xi\right] d \tau\right| \leq(M+1+0.5 d)|t-\bar{t}|
$$

for all $t, \bar{t} \in[0, \alpha]$. Thus, $T$ maps $U$ into itself. The continuity of $g$ shows that $T$ is continuous on $U$. Now, an application of the Schauder fixed point theorem yields the existence of at least one solution $\omega(\cdot, \varepsilon, \xi)$ of the equation $y=T[y]$. The solution is defined on $(-\infty, \alpha]$ for each $\varepsilon$ and $\xi$ satisfying (8) and (12) respectively. It is easily seen that $\omega(\cdot, \varepsilon, \xi)$ is a classical solution of (10), (11).

Now we prove that for $\xi_{1}<\xi_{2}$ satisfying (12) we have

$$
\omega\left(t, \varepsilon, \xi_{1}\right)<\omega\left(t, \varepsilon, \xi_{2}\right) \quad \text { for } t \in(0, \alpha] .
$$

Suppose not. Then the set

$$
I_{+}=\left\{t \in[0, \alpha]: \omega\left(t, \varepsilon, \xi_{1}\right) \geq \omega\left(t, \varepsilon, \xi_{2}\right)\right\}
$$

is not empty. If we put $\widetilde{t}=\min I_{+}$, it is clear that $\widetilde{t}>0$ and

$$
\omega^{\prime}\left(\widetilde{t}, \varepsilon, \xi_{1}\right) \geq \omega^{\prime}\left(\widetilde{t}, \varepsilon, \xi_{2}\right) \text {. }
$$

It follows from the monotonicity condition $W_{+}$and from (10) that

$$
\omega^{\prime}\left(t, \varepsilon, \xi_{1}\right)-\omega^{\prime}\left(t, \varepsilon, \xi_{2}\right)<g\left(\widetilde{t}, \omega_{\tilde{t}}^{\tau}\left(\cdot, \varepsilon, \xi_{1}\right)\right)-g\left(\widetilde{t}, \omega_{\tilde{t}}\left(\cdot, \varepsilon, \xi_{2}\right)\right) \leq 0,
$$

which contradicts (15). Hence $I_{+}$is empty and the statement follows.

Consider now a sequence $\left\{\xi_{m}\right\}_{m=0}^{\infty}$ of numbers such that

(i) $\xi_{m}$ satisfies (12) for $m \geq 0$,

(ii) $\xi_{m+1}<\xi_{m}$ for $m \geq 0$ and $\lim _{m \rightarrow \infty} \xi_{m}=0$.

Then the sequence $\left\{\omega\left(\cdot, \varepsilon, \xi_{m}\right)\right\}_{m=0}^{\infty}$ is uniformly convergent on $[0, \alpha]$ and

$$
\lim _{m \rightarrow \infty}\left\|\xi_{m} \chi\right\|_{X}=0 \text {. }
$$


Moreover we have

$$
\omega\left(t, \varepsilon, \xi_{m}\right)=\eta(0)+\varepsilon+\xi_{m}+\int_{0}^{t}\left[g\left(\tau, \omega_{\tau}\left(\cdot, \varepsilon, \xi_{m}\right)\right)+\varepsilon+\xi_{m}\right] d \tau
$$

for all $t \in[0, \alpha]$. Write

$$
\omega(t, \varepsilon)=\lim _{m \rightarrow \infty} \omega\left(t, \varepsilon, \xi_{m}\right), \quad t \in(-\infty, \alpha] .
$$

Letting $m \rightarrow \infty$ in (16) shows that $\omega(\cdot, \varepsilon)$ is a classical solution of (5), (6). It remains to show that it is the maximal solution on $(-\infty, \alpha]$. Let $\widetilde{\omega}(\cdot, \varepsilon)$ : $(-\infty, \widetilde{\alpha}] \rightarrow \mathbb{R}$ be a solution of $(5),(6)$ and $\alpha_{0}=\min (\alpha, \widetilde{\alpha})$. Then

$$
\begin{gathered}
\widetilde{\omega}^{\prime}(t, \varepsilon)<g\left(t, \omega_{t}(\cdot, \varepsilon)\right)+\varepsilon+\xi_{m}, \\
\omega^{\prime}\left(t, \varepsilon, \xi_{m}\right)=g\left(t, \omega_{t}\left(\cdot, \varepsilon, \xi_{m}\right)\right)+\varepsilon+\xi_{m}, \quad t \in\left[0, \alpha_{0}\right],
\end{gathered}
$$

and consequently $\widetilde{\omega}(t, \varepsilon)<\omega\left(t, \varepsilon, \xi_{m}\right)$ for $t \in\left[0, \alpha_{0}\right]$ and $m \geq 0$. It follows from $(17)$ that $\widetilde{\omega}(t, \varepsilon) \leq \omega(t, \varepsilon)$ for $t \in\left[0, \alpha_{0}\right]$. Thus $\omega(\cdot, \varepsilon)$ is the maximal solution of $(5),(6)$.

Let $\omega(\cdot)$ denote the maximal solution of $(3),(4)$. It follows easily that for $\varepsilon$ satisfying (8) we have

$$
\omega(t) \leq \omega(t, \varepsilon), \quad t \in[0, \alpha]
$$

The functions $\left\{\left.\omega(\cdot, \varepsilon)\right|_{[0, \alpha]}\right\}$ are uniformly bounded and equicontinuous. It follows from assumption (7) and from the Ascoli-Arzelà theorem that assertion (9) holds. This completes the proof.

REMARK 2.1. Suppose that $0 \leq \varepsilon \leq d / 4$ and

1) the function $\eta \mapsto\|\eta(\cdot \varepsilon)\|_{X}$ is nondecreasing,

2) $K<1$ and the constant $d$ satisfies the condition

$$
L\|\eta(\cdot, d / 4)\|_{X} \leq(1-K) d / 4 .
$$

Then the second inequality in (8) is satisfied.

Now we prove a theorem on the continuous dependence of maximal solutions of $(3)$, (4) on initial functions.

Assumption $H_{c}[X]$. Assumption $H[X]$ is fulfilled and if $y:(-\infty, a] \rightarrow \mathbb{R}$ is such that $y_{0} \in X$ and $\left.y\right|_{[0, a]} \in C([0, a], \mathbb{R})$ then the mapping $(\tau, t) \mapsto s_{t} y_{\tau}$ is continuous for $(\tau, t) \in[0, a] \times[0, a], t \geq \tau$.

THEOREM 2.2. Suppose that

1) Assumptions $H_{c}[X]$ and $H[\eta(\cdot, \varepsilon)]$ hold and $\eta \in X$,

2) condition 2) of Theorem 2.1 is satisfied and $\omega$ is the maximal solution of $(3),(4)$ existing on the interval $(-\infty, a]$. 
Then for any $0<a_{0}<a$ there is $\varepsilon_{0}>0$ such that for each $0<\varepsilon \leq \varepsilon_{0}$ the maximal solution $\omega(\cdot, \varepsilon)$ of problem $(5),(6)$ exists on $\left(-\infty, a_{0}\right]$ and

$$
\lim _{\varepsilon \rightarrow 0} \omega(t, \varepsilon)=\omega(t) \quad \text { uniformly on }\left[0, a_{0}\right] \text {. }
$$

Proof. Let $0<a_{0}<a$ be fixed. The proof will be divided into 2 steps.

I. We first prove the following property of equations with unbounded delay. Suppose that $\tau \in\left[0, a_{0}\right]$ and that the family of functions

$$
\gamma(\cdot, \varepsilon):(-\infty, \tau] \rightarrow \mathbb{R}_{+}, \quad \varepsilon>0
$$

satisfies the conditions

(i) $\gamma_{0}(\cdot, \varepsilon) \in X$ and $\gamma(\tau, \varepsilon)=\varepsilon$ for $\varepsilon \geq 0$,

(ii) $\left.\gamma(\cdot, \varepsilon)\right|_{[0, \tau]}$ is continuous for each $\varepsilon \geq 0$ and

$$
\lim _{\varepsilon \rightarrow 0}\left[\left\|\gamma_{0}(\cdot, \varepsilon)\right\|_{X}+\|\gamma(\cdot, \varepsilon)\|_{[0, \tau]}\right]=0 .
$$

Consider the Cauchy problem

$$
\begin{aligned}
y^{\prime}(t) & =g\left(t, y_{t}\right)+\varepsilon, \\
y(t) & =\omega(t)+\gamma(t, \varepsilon) \quad \text { for } t \in(-\infty, \tau] .
\end{aligned}
$$

We prove that there are $\widetilde{\varepsilon}>0$ and $\widetilde{\alpha}>0$ (independent of $\tau$ ) such that for $0<\varepsilon \leq \widetilde{\varepsilon}$ there is the maximal solution $\widetilde{\omega}(\cdot, \varepsilon, \tau)$ of (18), (19) defined on $(-\infty, \tau+\widetilde{\alpha}]$ and

$$
\lim _{\varepsilon \rightarrow 0} \widetilde{\omega}(t, \varepsilon, \tau)=\omega(t) \quad \text { uniformly on }[\tau, \tau+\widetilde{\alpha}] .
$$

It is easily seen that problem (18), (19) is equivalent to

$$
\begin{aligned}
y^{\prime}(t) & =g\left(t+\tau, y_{t}\right)+\varepsilon, \\
y(t) & =\omega_{\tau}(t)+\gamma_{\tau}(t, \varepsilon) \quad \text { for } t \in \mathbb{R}_{-} .
\end{aligned}
$$

More precisely, a function $\widetilde{y}:(-\infty, \tau+\widetilde{\alpha}] \rightarrow \mathbb{R}$ satisfies (18), (19) if and only if the function $\bar{y}:(-\infty, \tau] \rightarrow \mathbb{R}$ given by $\bar{y}(t)=\widetilde{y}(t+\tau)$ satisfies $(20)$, (21). It follows from Assumption $H_{c}[X]$ that there are $\widetilde{M}, \widetilde{b}>0$ such that

$$
\left|g\left(t+\tau, s_{t} \omega_{\tau}\right)\right| \leq \widetilde{M}, \quad \tau \in\left[0, a_{0}\right], t \in[\tau, a],
$$

and

$$
\left|g(t+\tau, w)-g\left(t+\tau, s_{t} \omega_{\tau}\right)\right| \leq 1 \quad \text { for }\left\|w-s_{t} \omega_{\tau}\right\|_{X} \leq \widetilde{b},
$$

for all $\tau \in\left(0, a_{0}\right]$ and $t \in[\tau, a]$. Suppose that $\varepsilon$ satisfies

$$
\begin{aligned}
& 0<\varepsilon \leq \widetilde{b} / 4, \\
& K \varepsilon+L\left\|\gamma_{0}(\cdot, \varepsilon)\right\|_{X}+K \max \{|\gamma(\xi, \varepsilon)|: \xi \in[0, \tau]\} \leq \widetilde{b} / 4 .
\end{aligned}
$$

Write

$$
\widetilde{\alpha}=\min \left\{a-a_{0}, \frac{\widetilde{b}}{2 K(\widetilde{M}+1+0.5 \widetilde{b})}\right\} .
$$


It follows from Theorem 2.1 that the maximal solution $\widetilde{\omega}(\cdot, \varepsilon, \tau)$ of $(20),(21)$ exists on $(-\infty, \widetilde{\alpha}]$ and

$$
\lim _{\varepsilon \rightarrow 0} \widetilde{\omega}(t, \varepsilon, \tau)=\omega_{\tau}(t) \quad \text { uniformly on }[0, \widetilde{\alpha}] \text {. }
$$

According to the above considerations, for $\varepsilon$ satisfying (22) there exists the maximal solution $\omega(\cdot, \varepsilon, \tau)$ of problem (18), (19). The solution is defined on $(-\infty, \tau+\widetilde{\alpha}]$ and

$$
\lim _{\varepsilon \rightarrow 0} \omega(t, \varepsilon, \tau)=\omega(t) \quad \text { uniformly on }[\tau, \tau+\widetilde{\alpha}] .
$$

II. Fix $\bar{\varepsilon}>0$. We will prove that there is $\widetilde{\varepsilon}>0$ such that for $0<\varepsilon \leq \widetilde{\varepsilon}$ we have:

(i) the maximal solution $\omega(\cdot, \varepsilon)$ of $(5)$, (6) exists on $\left(-\infty, a_{0}\right]$,

(ii) $\omega(t, \varepsilon)-\omega(t)<\bar{\varepsilon}$ for $t \in\left[0, a_{0}\right]$.

Consider the Cauchy problem (18), (19) with $\tau=0$ and $\gamma(\cdot, \varepsilon)=\eta(\cdot, \varepsilon)$ for $\varepsilon>0$. According to step I, we find $\varepsilon_{1}>0$ such that for $0<\varepsilon \leq \varepsilon_{1}$ there exists the maximal solution $\omega(\cdot, \varepsilon)$ of $(5),(6)$. The function $\omega(\cdot, \varepsilon)$ is defined on $(-\infty, \widetilde{\alpha}]$ and $\omega(t, \varepsilon)-\omega(t)<\bar{\varepsilon}$ for $t \in[0, \widetilde{\alpha}]$.

Now consider the functional differential problem

$$
\begin{aligned}
y^{\prime}(t) & =g\left(t, y_{t}\right)+\varepsilon, \\
y(t) & =\omega(t, \varepsilon) \quad \text { for } t \in(-\infty, \widetilde{\alpha}],
\end{aligned}
$$

where $0<\varepsilon<\varepsilon_{1}$. It follows that there is $\widetilde{\varepsilon}_{2}>0$ such that for $0<\varepsilon \leq \varepsilon_{2}$ we have:

(i) the maximal solution $\omega_{2}(\cdot, \varepsilon)$ of $(23),(24)$ is defined on $(-\infty, 2 \widetilde{\alpha}]$,

(ii) $\omega_{2}(t, \varepsilon)-\omega(t)<\bar{\varepsilon}$ for $t \in[\widetilde{\alpha}, 2 \widetilde{\alpha}]$.

Thus, the maximal solution $\omega(\cdot, \varepsilon)$ of $(5)$, (6) for $0<\varepsilon \leq \varepsilon_{2}$ is defined on $(-\infty, 2 \widetilde{\alpha}]$ and

$$
\omega(t, \varepsilon)-\omega(t)<\bar{\varepsilon} \quad \text { for } t \in[0,2 \widetilde{\alpha}] .
$$

The above procedure can be repeated $k$ times until $k \widetilde{\alpha}>a_{0}$, which gives the existence of $\varepsilon_{k}>0$ such that for any $0<\varepsilon \leq \varepsilon_{k}$, the maximal solution $\omega(\cdot, \varepsilon)$ of problem (5), (6) is defined on $\left(-\infty, a_{0}\right] \subset(-\infty, k \widetilde{\alpha}]$ and $\omega(t, \varepsilon)-\omega(t)<\bar{\varepsilon}$ for $t \in\left[0, a_{0}\right]$. This completes the proof.

3. Comparison theorems for mixed problems. We formulate assumptions on the space $Y$. Write $I=\left[0, r^{\prime}\right] \times\left[-r^{\prime \prime}, 0\right]$. Assume that $c>0$, $w:(-\infty, c] \times I \rightarrow \mathbb{R}$ and $t \in(-\infty, c]$. Define a function $w_{(t)}: D \rightarrow \mathbb{R}$ by $w_{(t)}(\tau, s)=w(t+\tau, s),(\tau, s) \in D$. If $w:(-\infty, c] \times I \rightarrow \mathbb{R}, c>0$, and $\left.w\right|_{[0, a] \times I} \in C([0, a] \times I, \mathbb{R})$ then we write

$$
\|w\|_{[0, t] \times I}=\max \{|w(\tau, s)|:(\tau, s) \in[0, t] \times I\}, \quad 0 \leq t \leq c .
$$

The axioms on $Y$ are the following. 
Assumption $H[Y] . \quad Y$ is a normed linear space with the norm $\|\cdot\|_{Y}$ which satisfies the conditions:

1) there is $\xi \in \mathbb{R}_{+}$such that for all $w \in Y$ we have

$$
|w(0, x)| \leq \xi\|w\|_{Y}, \quad x \in I,
$$

2) if $w:(-\infty, c] \times I \rightarrow \mathbb{R}, c>0$, is a function such that $w_{(0)} \in Y$ and $\left.w\right|_{[0, c] \times I} \in C([0, c] \times I, \mathbb{R})$ then $w_{(t)} \in Y$ for $t \in(0, c]$ and

(i) the mapping $t \mapsto w_{(t)}$ is continuous from $[0, c]$ to $Y$,

(ii) there are $K, K_{0} \in \mathbb{R}_{+}$independent of $w$ such that

$$
\left\|w_{(t)}\right\|_{Y} \leq K\|w\|_{[0, t] \times I}+K_{0}\left\|w_{(0)}\right\|_{Y}, \quad t \in[0, c] .
$$

3) $\left(Y,\|\cdot\|_{Y}\right)$ is a Banach space.

In order to get comparison results based on ordinary functional differential equations, it is necessary to formulate some relations between the spaces $Y$ and $X$.

For a function $w:(-\infty, 0] \times I \rightarrow \mathbb{R}$ we define $V[w]: \mathbb{R}_{-} \rightarrow \mathbb{R}_{+}$by

$$
V[w](t)=\sup \{|w(t, x)|: x \in I\}, \quad t \in \mathbb{R}_{-} ;
$$

the value $V[w](t)=\infty$ is not excluded. For $z: \Omega \rightarrow \mathbb{R}$ and $(t, x) \in E$ we write $V z_{(t, x)}$ instead of $V\left[z_{(t, x)}\right]$.

Assumption $H[X, Y]$. Assumptions $H_{c}[X]$ and $H[Y]$ hold and if $w \in Y$ then $V[w] \in X$.

Examples of spaces $Y$ and $X$ satisfying Assumption $H[X, Y]$ are given below. Note that Assumption $H[Y]$ and all the spaces $Y$ given in this section appear in the papers on the existence of solutions for equations with unbounded delay [9], [10].

EXAmple 3.1. Let $Y$ be the class of all functions $w: D \rightarrow \mathbb{R}$ which are uniformly continuous and bounded on $D$. For $w \in Y$ we put

$$
\|w\|_{Y}=\sup \{|w(\tau, s)|:(\tau, s) \in D\}
$$

Let $X$ be the space of all $\eta: \mathbb{R}_{-} \rightarrow \mathbb{R}$ which are uniformly continuous and bounded on $\mathbb{R}_{-}$. Write

$$
\|\eta\|_{X}=\sup \left\{|\eta(\tau)|: \tau \in \mathbb{R}_{-}\right\} .
$$

Then Assumption $H[X, Y]$ holds.

EXAmple 3.2. Let $Y$ be the class of all $w: D \rightarrow \mathbb{R}$ such that $w \in$ $C(D, \mathbb{R})$ and the limit

$$
\lim _{t \rightarrow-\infty} w(t, x)=w_{0}(x) \quad \text { exists uniformly with respect to } x \in I .
$$

The norm in $Y$ is defined by (25). Let $X$ be the space of all $\eta: \mathbb{R}_{-} \rightarrow \mathbb{R}$ such that $\eta \in C\left(\mathbb{R}_{-}, \mathbb{R}\right)$ and the $\operatorname{limit}_{\lim _{t \rightarrow-\infty}} \eta(t)$ exists. The norm in $X$ is defined by (26). Then Assumption $H[X, Y]$ is satisfied. 
EXAMPLE 3.3. Let $\gamma: \mathbb{R}_{-} \rightarrow(0, \infty)$ be a continuous nonincreasing function. Let $Y$ be the space of all continuous functions $w: D \rightarrow \mathbb{R}$ such that

$$
\lim _{t \rightarrow-\infty} w(t, x) / \gamma(t)=0, \quad x \in I .
$$

Put

$$
\|w\|_{Y}=\sup \{|w(t, x)| / \gamma(t):(t, x) \in D\} .
$$

Let $X$ be the class of all continuous functions $\eta: \mathbb{R}_{-} \rightarrow \mathbb{R}$ such that

$$
\lim _{t \rightarrow-\infty} \eta(t) / \gamma(t)=0
$$

Put

$$
\|\eta\|_{X}=\sup \left\{|\eta(t)| / \gamma(t): t \in \mathbb{R}_{-}\right\} .
$$

Then Assumption $H[X, Y]$ is satisfied.

Example 3.4. Fix $p \geq 1$. Denote by $Y$ the class of all $w: D \rightarrow \mathbb{R}$ such that

(i) for each $t \in \mathbb{R}_{-}$the function $w(t, \cdot): I \rightarrow \mathbb{R}$ is continuous,

(ii) for $x \in I$ we have

$$
\int_{-\infty}^{0}|w(\tau, x)|^{p} d \tau<\infty
$$

We define the norm in $Y$ by

$$
\begin{aligned}
\|w\|_{Y}= & \max \{|w(t, x)|:(t, x) \in\{0\} \times I\} \\
& +\int_{-\infty}^{0}\left[\max \left\{|w(\tau, x)|^{p}: x \in I\right\}\right] d \tau .
\end{aligned}
$$

Let $X$ be the space of all $\eta: \mathbb{R}_{-} \rightarrow \mathbb{R}$ such that

$$
\eta \text { is continuous at } 0 \text { and } \int_{-\infty}^{0}|\eta(\tau)|^{p} d \tau<\infty \text {. }
$$

Write

$$
\|\eta\|_{X}=\left(\int_{-\infty}^{0}|\eta(\tau)|^{p} d \tau\right)^{1 / p}
$$

Then Assumption $H[X, Y]$ holds.

EXAmple 3.5. Denote by $Y$ the set of all functions $w: D \rightarrow \mathbb{R}$ which are bounded and

(i) for each $t \in \mathbb{R}_{-}$the function $w(t, \cdot): I \rightarrow \mathbb{R}$ is continuous,

(ii) $w$ is continuous on $\{0\} \times I$ and for $x \in I$ we have

$$
W(x)=\sup \left\{\int_{-(m+1)}^{-m}|w(\tau, x)| d \tau: m \in \mathbb{N}\right\}<\infty .
$$


Here $\mathbb{N}$ is the set of natural numbers. The norm in $Y$ is defined by

$$
\|w\|_{Y}=\max \{|w(t, x)|:(t, x) \in\{0\} \times I\}+\sup \{W(x): x \in I\} .
$$

Let $X$ be the class of all $\eta: \mathbb{R}_{-} \rightarrow \mathbb{R}$ which are bounded and

$$
\|\eta\|_{0}=\sup \left\{\int_{-(m+1)}^{-m}|\eta(\tau)| d \tau: n \in \mathbb{N}\right\}<\infty .
$$

We also assume that $\eta$ is continuous at 0 . Write

$$
\|\eta\|_{X}=|\eta(0)|+\|\eta\|_{0}
$$

Then Assumption $H[X, Y]$ is satisfied.

Suppose that

$$
\sigma:[0, a] \times X_{+} \rightarrow \mathbb{R}_{+} \quad \text { and } \quad \lambda: E \rightarrow \mathbb{R}^{n}, \lambda=\left(\lambda_{1}, \ldots, \lambda_{n}\right),
$$

are given functions. We consider functional differential inequalities of the form

$$
\left|\partial_{t} z(t, x)-\sum_{i=1}^{n} \lambda_{i}(t, x) \partial_{x_{i}} z(t, x)\right| \leq \sigma\left(t, V z_{(t, x)}\right) .
$$

We prove that a function $u: \Omega \rightarrow \mathbb{R}$ satisfying (27) can be estimated by a maximal solution of a suitable initial value problem for an ordinary functional differential equation.

Assumption $H[\sigma, \lambda]$. The functions $\sigma \in C\left([0, a] \times X_{+}, \mathbb{R}_{+}\right)$and $\lambda \in$ $C\left(E, \mathbb{R}_{+}^{\kappa} \times \mathbb{R}_{-}^{n-\kappa}\right)$ satisfy the conditions:

1) $\sigma$ satisfies the monotonicity condition $W_{+}$,

2) for any function $\eta \in X_{+}$the maximal solution of the initial problem

$$
y^{\prime}(t)=\sigma\left(t, y_{t}\right), \quad y_{0}=\eta,
$$

is defined on $(-\infty, a]$.

We can now formulate the main result in this section.

Theorem 3.1. Suppose that Assumptions $H[X, Y]$ and $H[\sigma, \lambda]$ are satisfied and

1) $u: \Omega \rightarrow \mathbb{R}$ is continuous on $E \cup \partial_{0} E$ and $u_{(0, x)} \in Y$ for $x \in[-b, b]$,

2) there is $\eta \in X_{+}$such that $\eta(t) \leq \eta(0)$ for $t \in \mathbb{R}_{-}$and

$$
\begin{array}{ll}
|u(t, x)| \leq \eta(t) & \text { for }(t, x) \in E_{0}, \\
|u(t, x)| \leq \eta(0) & \text { for } \quad(t, x) \in \partial_{0} E,
\end{array}
$$

3) $u$ satisfies (27) on $E$.

Under the above assumptions we have

$$
|u(t, x)| \leq \omega(t, \eta) \quad \text { for }(t, x) \in E,
$$

where $\omega(\cdot, \eta)$ is the maximal solution of $(28)$. 
Proof. Define

$$
\widetilde{\omega}(t)=\sup \left\{|u(t, x)|: x \in\left[-b^{\prime}, c^{\prime}\right] \times\left[-c^{\prime \prime}, b^{\prime \prime}\right]\right\}, \quad t \in(-\infty, a] .
$$

Then estimate (29) is equivalent to

$$
\widetilde{\omega}(t) \leq \omega(t, \eta), \quad t \in(-\infty, a] .
$$

Fix $a_{0} \in(0, a)$. Consider the Cauchy problem

$$
\begin{aligned}
y^{\prime}(t) & =\sigma\left(t, y_{t}\right)+\varepsilon, \\
y(t) & =\eta(t)+h(t, \varepsilon), \quad t \in \mathbb{R}_{-},
\end{aligned}
$$

where

$$
h(t, \varepsilon)= \begin{cases}0 & \text { for } t \in(-\infty,-\varepsilon], \\ t+\varepsilon & \text { for } t \in(-\varepsilon, 0] .\end{cases}
$$

It follows that there is $\varepsilon_{0}>0$ such that for $0<\varepsilon \leq \varepsilon_{0}$ there is the maximal solution $\omega(\cdot, \eta, \varepsilon)$ of $(31),(32)$. The function $\omega(\cdot, \eta, \varepsilon)$ is defined on $\left(-\infty, a_{0}\right]$ and

$$
\lim _{\varepsilon \rightarrow 0} \omega(t, \eta, \varepsilon)=\omega(t, \eta) \quad \text { uniformly on }\left[0, a_{0}\right] .
$$

We prove that

$$
\widetilde{\omega}(t)<\omega(t, \eta, \varepsilon) \text { for } t \in\left[0, a_{0}\right],
$$

where $0<\varepsilon \leq \varepsilon_{0}$. Suppose this is not true. Then the set

$$
I_{+}=\left\{t \in\left[0, a_{0}\right]: \widetilde{\omega}(t) \geq \omega(t, \eta, \varepsilon)\right\}
$$

is not empty. If we put $\tilde{t}=\min I_{+}$it is clear that $\tilde{t}>0$ and

$$
\begin{aligned}
& \widetilde{\omega}(\tau)<\omega(\tau, \eta, \varepsilon) \quad \text { for } \tau \in[0, \widetilde{t}), \\
& \widetilde{\omega}(\widetilde{t})=\omega(\widetilde{t}, \eta, \varepsilon) .
\end{aligned}
$$

This gives

$$
D \_\widetilde{\omega}(\widetilde{t}) \geq \omega^{\prime}(\widetilde{t}, \eta, \varepsilon)
$$

where $D_{-}$is the left-hand lower Dini derivative. There is $\widetilde{x} \in\left[-b^{\prime}, c^{\prime}\right] \times$ $\left[-c^{\prime \prime}, b^{\prime \prime}\right], \widetilde{x}=\left(\widetilde{x}_{1}, \ldots, \widetilde{x}_{n}\right)$, such that $\widetilde{\omega}(\widetilde{t})=|u(\widetilde{t}, \widetilde{x})|$. Let $I^{(+)}, I^{(-)}, I^{(0)}$ be the sets of integers (possibly empty) such that $I^{(+)} \cup I^{(-)} \cup I^{(0)}=\{1, \ldots, n\}$ and

$$
\begin{gathered}
\widetilde{x}_{i} \geq b_{i} \quad \text { for } i \in I^{(+)}, \quad \widetilde{x}_{i} \leq-b_{i} \quad \text { for } i \in I^{(-)}, \\
-b_{i}<\widetilde{x}_{i}<b_{i} \quad \text { for } i \in I^{(0)}
\end{gathered}
$$

Since $(\widetilde{t}, \widetilde{x}) \notin \partial_{0} E$, we have

$$
\{1, \ldots, \kappa\} \cap I^{(+)}=\emptyset \quad \text { and } \quad\{\kappa+1, \ldots, n\} \cap I^{(-)}=\emptyset .
$$


It follows that either (i) $\widetilde{\omega}(\widetilde{t})=u(\widetilde{t}, \widetilde{x})$ or (ii) $\widetilde{\omega}(\widetilde{t})=-u(\widetilde{t}, \widetilde{x})$. If (i) holds, then

$$
\begin{array}{cl}
\partial_{x_{i}} u(\widetilde{t}, \widetilde{x}) \geq 0 \quad \text { for } i \in I^{(+)}, \quad \partial_{x_{i}} u(\widetilde{t}, \widetilde{x}) \leq 0 \quad \text { for } i \in I^{(-)}, \\
& \partial_{x_{i}} u(\widetilde{t}, \widetilde{x})=0 \quad \text { for } i \in I^{(0)} .
\end{array}
$$

It follows from Assumption $H[\sigma, \lambda]$ and from (27) that

$$
\begin{aligned}
D_{-} \widetilde{\omega}(\widetilde{t}) & \leq \partial_{t} u(\widetilde{t}, \widetilde{x}) \leq \sigma\left(\widetilde{t}, V u_{(\widetilde{t}, \widetilde{x})}\right)+\sum_{i=1}^{n} \lambda_{i}(\widetilde{t}, \widetilde{x}) \partial_{x_{i}} u(\widetilde{t}, \widetilde{x}) \\
& <\sigma\left(\widetilde{t}, \omega_{\tilde{t}}(\cdot, \eta, \varepsilon)\right)+\varepsilon=\omega^{\prime}(\widetilde{t}, \eta, \varepsilon),
\end{aligned}
$$

which contradicts (35).

Similar considerations apply if case (ii) holds. Then the set $I_{+}$is empty and estimate (34) follows.

Letting $\varepsilon$ tend to 0 in (34), we obtain inequality $(30)$ on $\left[0, a_{0}\right]$. By the arbitrariness of $a_{0} \in(0, a)$, the assertion follows.

REMARK 3.1. Assumption 2) of Theorem 3.1 can be replaced by

$\left.2^{\prime}\right)$ there is $\eta \in X_{+}$such that $|u(t, x)| \leq \eta(t)$ for $(t, x) \in E_{0}$ and $|u(t, x)| \leq \omega(t, \eta)$ for $(t, x) \in \partial_{0} E$ where $\omega(\cdot, \eta)$ is the maximal solution of (28).

Let us now consider two problems: problem (1), (2) and the following one:

$$
\begin{aligned}
\partial_{t} z(t, x) & =\widetilde{f}\left(t, x, z_{(t, x)}, \partial_{x} z(t, x)\right), \\
z(t, x) & =\widetilde{\varphi}(t, x) \quad \text { for }(t, x) \in E_{0} \cup \partial_{0} E,
\end{aligned}
$$

where $\tilde{f}: E \times Y \times \mathbb{R}^{n} \rightarrow \mathbb{R}$ and $\widetilde{\varphi}: E_{0} \cup \partial_{0} E \rightarrow \mathbb{R}$ are given functions. Let $u, \widetilde{u}: \Omega \rightarrow \mathbb{R}$ denote classical solutions of (1), (2) and (36), (37) respectively. Now we are going to give an estimate of $u-\widetilde{u}$ on $E$.

Assumption $H[f]$. Assumption $H[X, Y]$ is satisfied and

1) the function $f: E \times Y \times \mathbb{R}^{n} \rightarrow \mathbb{R}$ of the variables $(t, x, w, q)$ is continuous and the derivatives $\left(\partial_{q_{1}} f, \ldots, \partial_{q_{n}} f\right)=\partial_{q} f$ exist on $E \times Y \times \mathbb{R}^{n}$ and $\partial_{q} f \in C\left(E \times Y \times \mathbb{R}^{n}, \mathbb{R}^{n}\right)$,

2) for $(t, x, w, q) \in E \times Y \times \mathbb{R}^{n}$ we have

$$
\begin{array}{ll}
\partial_{q_{i}} f(t, x, w, q) \geq 0 & \text { for } 1 \leq i \leq \kappa, \\
\partial_{q_{i}} f(t, x, w, q) \leq 0 & \text { for } \kappa+1 \leq i \leq n,
\end{array}
$$

3) there is $\sigma \in C\left([0, a] \times X_{+}, \mathbb{R}_{+}\right)$such that

(i) $\sigma$ satisfies the monotonicity condition $W_{+}$and for each $\eta \in X_{+}$ and $\gamma \in C\left([0, a], \mathbb{R}_{+}\right)$the maximal solution of the problem 


$$
y^{\prime}(t)=\sigma\left(t, y_{t}\right)+\gamma(t), y_{0}=\eta,
$$

exists on $(-\infty, a]$

(ii) the estimate

$$
|f(t, x, w, q)-f(t, x, \bar{w}, q)| \leq \sigma(t, V[w-\bar{w}])
$$

is satisfied on $E \times Y \times \mathbb{R}^{n}$.

TheOREm 3.2. Suppose that Assumption $H[f]$ is satisfied and

1) the functions $\varphi, \widetilde{\varphi}: E_{0} \cup \partial_{0} E \rightarrow \mathbb{R}$ are continuous on $\partial_{0} E$ and satisfy $\varphi_{(0, x)} \in Y, \widetilde{\varphi}_{(0, x)} \in Y$ for $x \in[-b, b]$,

2) there is $\eta \in X_{+}$such that $\eta(t) \leq \eta(0)$ for $t \in \mathbb{R}_{-}$and

$$
\begin{array}{ll}
|\varphi(t, x)-\widetilde{\varphi}(t, x)| \leq \eta(t) \quad \text { for } \quad(t, x) \in E_{0}, \\
|\varphi(t, x)-\widetilde{\varphi}(t, x)| \leq \eta(0) \quad \text { for } \quad(t, x) \in \partial_{0} E,
\end{array}
$$

3) $\tilde{f}: E \times Y \times \mathbb{R}^{n} \rightarrow \mathbb{R}$ is continuous and there is $\gamma \in C\left([0, a], \mathbb{R}_{+}\right)$such that

$$
|f(t, x, w, q)-\widetilde{f}(t, x, w, q)| \leq \gamma(t) \quad \text { on } E \times Y \times \mathbb{R}^{n},
$$

4) $u, \widetilde{u}: \Omega \rightarrow \mathbb{R}^{n}$ are classical solutions of (1), (2) and (36), (37), respectively.

Under these assumptions we have

$$
|u(t, x)-\widetilde{u}(t, x)| \leq \omega(t, \eta, \gamma) \quad \text { for }(t, x) \in E,
$$

where $\omega(\cdot, \eta, \gamma)$ is the maximal solution of (38).

Proof. The function $\widetilde{z}(t, x)=(u-\widetilde{u})(t, x)$ for $(t, x) \in \Omega$ satisfies the initial boundary estimates

$$
\begin{array}{ll}
|\widetilde{z}(t, x)| \leq \eta(t) & \text { for }(t, x) \in E_{0}, \\
|\widetilde{z}(t, x)| \leq \eta(0) & \text { for }(t, x) \in \partial_{0} E .
\end{array}
$$

Moreover,

$$
\left|\partial_{t} \widetilde{z}(t, x)-\sum_{i=1}^{n} \int_{0}^{1} \partial_{q_{i}} f(P(t, x, \tau)) d \tau \partial_{x_{i}} \widetilde{z}(t, x)\right| \leq \sigma\left(t, V \widetilde{z}_{(t, x)}\right)+\gamma(t),
$$

where $(t, x) \in E$ and

$$
P(t, x, \tau)=\left(t, x, u_{(t, x)}, \partial_{x} u(t, x)+\tau \partial_{x}(\widetilde{u}-u)(t, x)\right) .
$$

Hence, (39) follows by applying Theorem 3.1 to $\widetilde{z}$.

The following uniqueness and continuous dependence results are immediate consequences of Theorem 3.2.

Theorem 3.3. Suppose that Assumption $H[f]$ is satisfied and the function $y(t)=0, t \in(-\infty, a]$, is the maximal solution of (28) corresponding 
to $\eta(t)=0, t \in \mathbb{R}_{-}$. Then the mixed problem (1), (2) admits at most one classical solution. Moreover, for every $\varepsilon>0$ there exists $\delta>0$ such that if

$$
|f(t, x, w, q)-\tilde{f}(t, x, w, q)|<\delta \quad \text { on } E \times Y \times \mathbb{R}^{n}
$$

and

then

$$
|\varphi(t, x)-\widetilde{\varphi}(t, x)|<\delta \quad \text { on } E_{0} \cup \partial_{0} E
$$

$$
|u(t, x)-\widetilde{u}(t, x)|<\varepsilon \quad \text { on } E,
$$

where $u$ and $\widetilde{u}$ are the solutions of (1), (2) and (36), (37), respectively.

Proof. Uniqueness of classical solutions of (1), (2) is a consequence of Theorem 3.2 with $\tilde{f}=f, \gamma(t)=0$ for $t \in[0, a]$ and $\eta(t)=0$ for $t \in \mathbb{R}_{-}$.

For $\varepsilon>0$ we can choose $\delta>0$ so that the maximal solution $\omega(\cdot, \eta, \gamma)$ of (38), where $\gamma \in C\left([0, a], \mathbb{R}_{+}\right), \eta \in X_{+}$and $\gamma(t) \leq \delta$ on $[0, a], \eta(t) \leq \eta(0)=\delta$ for $t \in \mathbb{R}_{-}$, satisfies the condition $\omega(t, \eta, \gamma)<\varepsilon$ for $t \in[0, a]$. Now, assertion (40) follows from Theorem 3.2.

4. Functional differential inequalities. We now prove a theorem on weak functional differential inequalities generated by the mixed problem (1), (2).

Let $\theta: E \rightarrow \mathbb{R}^{n}$ be the zero function.

Assumption $H_{0}[f]$. Assumption $H[X, Y]$ is satisfied and

1) conditions 1), 2) of Assumption $H[f]$ hold,

2) $f$ satisfies the following monotonicity condition: if $w, \bar{w} \in Y$ and $w(\tau, s) \leq \bar{w}(\tau, s)$ for $(\tau, s) \in D$ and $w(0,0)=\bar{w}(0,0)$ then $f(t, x, w, q)$ $\leq f(t, x, \bar{w}, q)$ for all $(t, x, q) \in E \times \mathbb{R}^{n}$,

3) there exists $\sigma$ such that $H[\sigma, \theta]$ holds with $\bar{y}(t) \equiv 0$ being the maximal solution of $(28)$ for $\eta(t)=0, t \in \mathbb{R}_{-}$, and the estimate

$$
f(t, x, w, q)-f(t, x, \bar{w}, q) \leq \sigma(t, V[w-\bar{w}])
$$

is satisfied for $(t, x, q) \in E \times \mathbb{R}^{n}$ and for $w, \bar{w} \in Y$ such that $w(\tau, s) \geq$ $\bar{w}(\tau, s)$ on $D$.

Denote by $F$ the Nemytskil operator corresponding to (1), i.e.

$$
F[z](t, x)=f\left(t, x, z_{(t, x)}, \partial_{x} z(t, x)\right) .
$$

THEOREM 4.1. Suppose that Assumption $H_{0}[f]$ is satisfied and the functions $u, v: \Omega \rightarrow \mathbb{R}$ are such that

1) $\left.u\right|_{E \cup \partial_{0} E}$ and $\left.v\right|_{E \cup \partial_{0} E}$ are continuous and the partial derivatives

$$
\partial_{t} u(t, x), \partial_{t} v(t, x), \partial_{x} u(t, x), \partial_{x} v(t, x)
$$

exist on $E$, 
2) $u_{(0, x)}, v_{(0, x)} \in Y$ for $x \in[-b, b]$ and the initial boundary inequality

$$
u(t, x) \leq v(t, x), \quad(t, x) \in E_{0} \cup \partial_{0} E,
$$

is satisfied,

3) the differential functional inequality

$$
\partial_{t} u(t, x)-F[u](t, x] \leq \partial_{t} v(t, x)-F[v](t, x)
$$

holds on E.

Under these assumptions we have $u(t, x) \leq v(t, x)$ on $E$.

Proof. Fix $a_{0} \in(0, a)$. We will prove that

$$
u(t, x) \leq v(t, x) \quad \text { for }(t, x) \in E \cap\left(\left[0, a_{0}\right] \times \mathbb{R}^{n}\right) .
$$

Consider the Cauchy problem

$$
y^{\prime}(t)=\sigma\left(t, y_{t}\right)+\varepsilon, \quad y(t)=h(t, \varepsilon) \quad \text { for } t \in \mathbb{R}_{-},
$$

where $h(\cdot, \varepsilon)$ is given by (33). It follows that there is $\varepsilon_{0}>0$ such that for $0<\varepsilon \leq \varepsilon_{0}$ there is the maximal solution $\widetilde{\omega}(\cdot, \varepsilon)$ of $(44)$. The function $\widetilde{\omega}(\cdot, \varepsilon)$ is defined on $\left(-\infty, a_{0}\right]$ and

$$
\lim _{\varepsilon \rightarrow 0} \widetilde{\omega}(t, \varepsilon)=0 \quad \text { uniformly on }\left[0, a_{0}\right] \text {. }
$$

Write

$$
\left.\widetilde{v}(t, x)=v(t, x)+\widetilde{\omega}(t, \varepsilon) \quad \text { for }(t, x) \in \Omega \cap\left(\left(-\infty, a_{0}\right] \times \mathbb{R}^{n}\right]\right) .
$$

We prove that

$$
u(t, x)<v(t, x) \quad \text { for }(t, x) \in E \cap\left(\left[0, a_{0}\right] \times \mathbb{R}^{n}\right) .
$$

Suppose otherwise. Then the set

$$
J_{+}=\left\{t \in\left[0, a_{0}\right]:(u-\widetilde{v})(t, x) \geq 0 \text { for some } x \in[-b, b]\right\}
$$

is not empty. If we put $\widetilde{t}=\min J_{+}$, it is clear that $\widetilde{t}>0$ and there exists $\widetilde{x}=\left(\widetilde{x}_{1}, \ldots, \widetilde{x}_{n}\right) \in[-b, b]$ such that

$$
u(t, x)<\widetilde{v}(t, x) \quad \text { for }(t, x) \in[0, \widetilde{t}) \times \mathbb{R}^{n}
$$

and $u(\widetilde{t}, \widetilde{x})=\widetilde{v}(\widetilde{t}, \widetilde{x})$. Then we have

$$
u_{(\widetilde{t}, \widetilde{x})}(\tau, s) \leq \widetilde{v}_{(\vec{t}, \widetilde{x}}(\tau, s) \quad \text { for }(\tau, s) \in D, \quad u_{(\widetilde{t}, \widetilde{x})}(0,0)=\widetilde{v}_{(\widetilde{t}, \widetilde{x})}(0,0) .
$$

It is easy to see that

$$
\partial_{t}(u-\widetilde{v})(\widetilde{t}, \widetilde{x}) \geq 0
$$

There exist sets of integers $I^{(+)}, I^{(-)}, I^{(0)}$ (possibly empty) such that $I^{(+)} \cup$ $I^{(-)} \cup I^{(0)}=\{1, \ldots, n\}$ and

$$
\begin{gathered}
\widetilde{x}_{i}=b_{i} \quad \text { for } i \in I^{(+)}, \quad \widetilde{x}_{i}=-b_{i} \quad \text { for } i \in I^{(-)}, \\
-b_{i}<\widetilde{x}_{i}<b_{i} \text { for } i \in I^{(0)} .
\end{gathered}
$$


Since $(\widetilde{t}, \widetilde{x}) \notin \partial_{0} E$ we have $\{1, \ldots, \kappa\} \cap I^{(+)}=\emptyset$ and $\{\kappa+1, \ldots, n\} \cap I^{(-)}=\emptyset$. We thus get

$$
\partial_{x_{i}}(u-\widetilde{v})(\widetilde{t}, \widetilde{x}) \geq 0 \quad \text { for } i \in I^{(+)}, \quad \partial_{x_{i}}(u-\widetilde{v})(\widetilde{t}, \widetilde{x}) \leq 0 \quad \text { for } i \in I^{(-)}
$$

and

$$
\partial_{x_{i}}(u-\widetilde{v})(\widetilde{t}, \widetilde{x})=0 \quad \text { for } i \in I^{(0)} .
$$

From (41), (42), (46) it follows that

$$
\begin{aligned}
\partial_{t}(u-\widetilde{v})(\widetilde{t}, \widetilde{x}) \leq & F[u](\widetilde{t}, \widetilde{x})-F[v](\widetilde{t}, \widetilde{x})-\widetilde{\omega}^{\prime}(\widetilde{t}, \varepsilon) \\
\leq & {\left[f\left(\widetilde{t}, \widetilde{x}, \widetilde{v}_{(\widetilde{t}, \widetilde{x})}, \partial_{x} u(\widetilde{t}, \widetilde{x})\right)-f\left(\widetilde{t}, \widetilde{x}, v_{(\widetilde{t}, \widetilde{x})}, \partial_{x} u(\widetilde{t}, \widetilde{x})\right)\right] } \\
& +f\left(\widetilde{t}, \widetilde{x}, v_{(\widetilde{t}, \widetilde{x})}, \partial_{x} u(\widetilde{t}, \widetilde{x})\right) \\
& -f\left(\widetilde{t}, \widetilde{x}, v_{(\widetilde{t}, \widetilde{x})}, \partial_{x} \widetilde{v}(\widetilde{t}, \widetilde{x})\right)-\widetilde{\omega}^{\prime}(\widetilde{t}, \varepsilon) \\
\leq & \sigma\left(\widetilde{t}, \widetilde{\omega}_{\widetilde{t}}(\cdot, \varepsilon)\right)+\sum_{i=1}^{n} \partial_{q_{i}} f(Q) \partial_{x_{i}}(u-\widetilde{v})(\widetilde{t}, \widetilde{x})-\widetilde{\omega}^{\prime}(\widetilde{t}, \varepsilon),
\end{aligned}
$$

where $Q \in E \times Y \times \mathbb{R}^{n}$ is an intermediate point. From the above estimates and from $(48)$, (49) we deduce that $\partial_{t}(u-\widetilde{v})(\widetilde{t}, \widetilde{x})<0$, which contradicts (47). Thus estimate (45) is proved. Now, letting $\varepsilon \rightarrow 0$ we obtain (43). By the arbitrariness of $a_{0} \in(0, a)$ the assertion follows.

REMARK 4.1. Assumption 3) of Theorem 4.1 can be replaced by the following condition: the differential functional inequality (41) is satisfied on the set

$$
\{(t, x) \in E: u(t, x)>v(t, x)\} .
$$

Remark 4.2. Suppose that Assumption $H_{0}[f]$ is satisfied. Then the mixed problem (1), (2) admits at most one classical solution.

\section{References}

[1] A. Augustynowicz and Z. Kamont, On Kamke's functions in uniqueness theorems for first order partial differential-functional equations, Nonlinear Anal. 14 (1990), 837-850.

[2] P. Besala, On solutions of first order partial differential equations defined in an unbounded zone, Bull. Acad. Polon. Sci. Sér. Sci. Math. Astronom. Phys. 12 (1964), 95-99.

[3] - On the uniqueness of initial value problems for partial differential equations of first order, Ann. Polon. Math. 40 (1983), 105-108.

[4] P. Brandi, Z. Kamont and A. Salvadori, Differential and differential difference inequalities related to mixed problems for first order partial differential functional equations, Atti Sem. Mat. Fis. Univ. Modena 39 (1991), 255-276.

[5] P. Brandi and C. Marcelli, Haar inequality in hereditary setting and applications, Rend. Sem. Mat. Univ. Padova 96 (1996), 177-194. 
[6] A. Haar, Sur l'unicité des solutions des équations aux dérivées partielles, C. R. Acad. Sci. Paris 187 (1928), 23-26.

[7] Y. Hino, S. Murakami and T. Naito, Functional-Differential Equations with Infinite Delay, Lecture Notes in Math. 1473, Springer, Berlin, 1991.

[8] Z. Kamont, Hyperbolic Functional Differential Inequalities and Applications, Kluwer, Dordrecht, 1999.

[9] Z. Kamont and S. Kozieł, First order partial functional differential equations with unbounded delay, Georgian Math. J. 10 (2003), 509-530.

[10] - - - Mixed problems for hyperbolic functional differential equations with unbounded delay, Nonlinear Anal. 58 (2004), 489-515.

[11] Z. Kamont and H. Leszczyński, Uniqueness result for generalized entropy solutions to the Cauchy problem for first order partial differential functional equations, Z. Anal. Anwend. 13 (1994), 477-491.

[12] S. N. Kruzhkov, Generalized solutions of nonlinear first order partial differential equations, Mat. Sb. 70 (1966), 394-415 (in Russian).

[13] V. Lakshmikantham and S. Leela, Differential and Integral Inequalities, Academic Press, New York and London, 1969.

[14] J. Szarski, Differential Inequalities, Polish Sci. Publ., Warszawa, 1967.

[15] - Comparison theorems for infinite systems of differential functional equations and strongly coupled infinite systems of first order partial differential equations, Rocky Mountain J. Math. 10 (1980), 237-246.

[16] K. Topolski, Classical methods for viscosity solutions of differential-functional inequalities, Nonlinear World 4 (1997), 1-18.

[17] T. D. Van, L. V. Hap and N. D. Thai Son, On some differential inequalities and the uniqueness of global semiclassical solutions to the Cauchy problem for weakly-coupled systems, J. Inequal. Appl. 2 (1998), 357-372.

[18] T. D. Van and N. D. Thai Son, Uniqueness of the global semiclassical solution to the Cauchy problem for first-order nonlinear partial differential equations, Diff. Equations 30 (1994), 659-666.

[19] T. D. Van, M. Tsuji and N. D. Thai Son, The Characteristic Method and its Generalizations for First-Order Nonlinear Partial Differential Equations, Chapman \& Hall/CRC, Boca Raton, FL, 2000.

[20] T. Ważewski, Sur l'unicité et la limitation des intégrales des équations aux derivées partielles du premier ordre, Rend. Accad. Lincei 17 (1933), 372-376.

Z. Kamont

Institute of Mathematics

University of Gdańsk

Wit Stwosz St. 57

80-952 Gdańsk, Poland

E-mail: zkamont@math.univ.gda.pl
S. Kozieł

Faculty of Electr. Telecomm. and Inf. Technical University of Gdańsk Narutowicz St. 11-12 80-952 Gdańsk, Poland E-mail: koziel@ue.eti.pg.gda.pl

Received 23.3.2005

and in final form 14.11.2005 